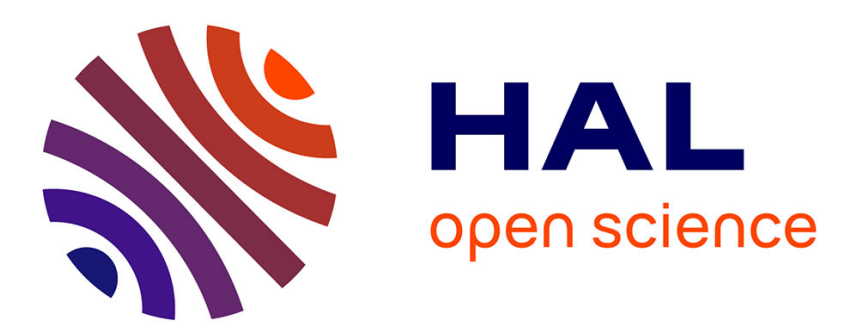

\title{
La participation des salariés: Une expression du don dans les organisations
}

\author{
Nicolas Aubert
}

\section{To cite this version:}

Nicolas Aubert. La participation des salariés: Une expression du don dans les organisations. RIMHE:

Revue Interdisciplinaire Management, Homme(s) \& Entreprise, 2016, 10.3917/rimhe.021.0069 . halshs-01299098v2

\section{HAL Id: halshs-01299098 \\ https://shs.hal.science/halshs-01299098v2}

Submitted on 31 Dec 2018

HAL is a multi-disciplinary open access archive for the deposit and dissemination of scientific research documents, whether they are published or not. The documents may come from teaching and research institutions in France or abroad, or from public or private research centers.
L'archive ouverte pluridisciplinaire HAL, est destinée au dépôt et à la diffusion de documents scientifiques de niveau recherche, publiés ou non, émanant des établissements d'enseignement et de recherche français ou étrangers, des laboratoires publics ou privés. 


\title{
La participation des salariés : Une expression du don dans les organisations ${ }^{1}$
}

\author{
Nicolas AUBERT ${ }^{2}$
}

Résumé : Pour l'économiste François Perroux, la contrainte, l'échange et le don sont les trois motifs des actes économiques. Son originalité est d'analyser la participation à la lumière du dernier motif. Il conçoit la participation comme un don à l'œuvre commune et adopte une vision de la participation ne se limitant pas au seul partage des bénéfices. Sa critique de l'échange libéral et de la contrainte marxiste traduit les angoisses de son temps mais les formes contemporaines de l'échange et de la contrainte rendent actuelles ses analyses. Rapprocher don et participation trouve sa cohérence dans des travaux qui se focalisent sur la participation financière des salariés. Les travaux empiriques les plus récents montrent en effet la pertinence des théories du don et de la réciprocité pour comprendre la participation des salariés.

Mots-clés : Participation des salariés, participation financière des salariés, participation des salariés à la gestion, don, échange, contrainte.

\footnotetext{
${ }^{1}$ Nous remercions la rédactrice en chef et deux relecteurs anonymes pour leurs commentaires.

${ }^{2}$ Professeur des Universités, Aix-Marseille Université, CERGAM (EA 4225), niaubert@ gmail.com.
} 


\title{
Employees' participation:
}

\section{An expression of gift within organizations}

\begin{abstract}
According to the French economist François Perroux, constraint, exchange and gift are the three motives of economic actions. Its originality is to analyze participation in the light of the last motive. He regards participation as a gift to the common work and adopt a vision of participation that is not restricted solely to its financial components. His critique of liberal exchange and Marxist constraint reflects the anxieties of his time but the contemporary forms of exchange and constraint still make his point valid. Bringing together gift and participation finds its coherence in works focusing on employee financial participation. The most recent empirical studies show the relevance of gift and reciprocity theories to understand employees' participation.
\end{abstract}

Keywords: Employees' participation, employees' financial participation, employees' participation in decision making, gift, exchange, constraint. 


\section{Introduction}

Le huitième alinéa de la Constitution de 1946 stipule que: "Tout travailleur participe, par l'intermédiaire de ses délégués, à la détermination collective des conditions de travail ainsi qu'à la gestion des entreprises ». Faire de la participation un principe constitutionnel révèle l'importance de ce thème en France. Une importance réaffirmée à chaque rapport parlementaire ou loi touchant à la participation. En France, les principaux dispositifs ont été mis au point durant le mandat présidentiel du général De Gaulle. Dans un discours de 1969, il expliquait: "La participation est la grande réforme de notre siècle! » (cité par Guiol, 2009). Dans le même discours de 1969, il énonçait « ce qui est en cause, c'est la condition de l'homme. Il s'agit donc, partout où les hommes sont ensemble pour vivre et pour travailler, de rendre leurs rapports plus humains, plus dignes, par-là plus efficaces. Il s'agit que chacun, là où il fournit son effort, ne soit pas un instrument passif mais participe activement à son groupe de destin. (...) Ce qui s'impose au total, c'est la Participation, réforme qui est, assurément, de longue haleine et de grande envergure. »

La France est dotée de plusieurs dispositifs de participation des salariés. Si l'on associe le plus souvent ces dispositifs à de Gaulle, c'est qu'il en fut le principal instigateur. La participation aux bénéfices, l'intéressement et l'actionnariat salarié sont désormais les formes les plus populaires de la participation financière. Ceux qui aujourd'hui souhaitent réformer ou améliorer les systèmes de participation se réfèrent encore au général de Gaulle. On lui associe donc fréquemment l'idée de participation en France. Cette impulsion gaullienne fut précédée par d'autres faits marquants. Dès la libération, une ordonnance du Conseil National de la Résistance du 22 février 1945 crée les comités d'entreprise en liant explicitement libération nationale et libération sociale. Il s'agit «d'associer les travailleurs à la gestion de leur entreprise et à la direction de l'économie ». Les expériences et initiatives participatives remontent au XIX ${ }^{\text {ème }}$ siècle $^{3}$. Elles résultent de la mise en œuvre des idées des socialistes français ou d'expériences de patrons imprégnés de la doctrine sociale de l'Eglise dont De Gaulle s'inspirait. La participation recouvre des réalités très différentes qui dépassent l'entreprise. Si l'on s'en tient toutefois à la participation dans l'entreprise, elle concerne avant tout la participation des salariés à ses bénéfices et à sa gestion. Les premières initiatives instauraient des systèmes de participation aux bénéfices faute de n'avoir pu obtenir un consensus au sein même de la famille gaulliste. Les gaullistes se divisaient déjà sur l'étendue de la participation (Godfrain, 2000 et Chatriot, 2012). Il semble en effet que la participation financière eut été envisagée comme un premier volet d'une participation élargie des salariés aux bénéfices et à la gestion que de Gaulle n'aurait pas eu le temps de mettre en œuvre. Mais les gaullistes peinaient néanmoins à construire une doctrine politique autour de la participation. Louis Vallon ${ }^{4}$ et René Capitant, les plus ardents défenseurs de la participation au sein de la famille gaulliste s'enthousiasmèrent à la lecture de l'ouvrage de Marcel

\footnotetext{
${ }^{3}$ Allocution radiodiffusée et télévisée au palais de l'Elysée, 11 mars 1969, Discours et Messages, V, pp. 386387. Allocution reprise par Dezes (1990, p. 105).

${ }^{4}$ Louis Vallon est l'auteur de l'amendement dit « Vallon» à la loi de finances 1965-1966 qui reconnaissait aux salariés le droit aux accroissements d'actifs dégagés par les bilans des entreprises.
} 
Loichot (1966) intitulé : «le pancapitalisme » dont ils pensaient qu'il comblait le manque de doctrine politique. Le pancapitalisme proposait que tous les travailleurs deviennent des capitalistes en recevant une partie de l'accroissement de la richesse de leur entreprise sous la forme d'actions. Godfrain (2000) pense qu'une telle réforme aurait permis aux salariés de devenir mécaniquement majoritaires au capital de leur entreprise en vingt ans du fait de la croissance économique de l'époque. Participation financière et participation à la gestion s'en seraient ainsi trouvées réconciliées. Chatriot (2012) rappelle que ces propositions rencontrèrent une vive opposition de la part du patronat et des syndicats qui toléraient la participation aux bénéfices mais pas la participation à la gestion. Il s'avéra que le pancapitalisme ne fondait pas une théorie de la participation mais proposait des recettes pour mettre en œuvre la participation. Le projet gaullien ne réalisa donc pas ses ambitions et se cantonna finalement à la participation aux bénéfices.

Aujourd'hui, de quelles théories disposons-nous pour comprendre la participation?

Force est de constater que la mise en œuvre de la participation aux bénéfices répond aux préoccupations de la théorie de l'agence issue de l'économie néoclassique qui forme toujours le courant dominant en gouvernement et finance d'entreprise. Ces théories analysent l'entreprise du point de vue des incitations - financières essentiellement - et excluent la participation à la gestion des salariés. Elles s'appuient sur une analyse des phénomènes économiques fondée sur les échanges marchands.

L'économiste François Perroux propose une autre conception de la participation. Il propose d'analyser la participation en la faisant reposer sur le don et non sur l'échange marchand. A l'époque où Perroux écrit, il s'agit de proposer une troisième voie entre libéralisme et marxisme. Il se concentre pour cela davantage sur l'identification des limites de la contrainte marxiste et de l'échange libéral comme motifs des actes économiques. Cette explication de la participation reposant sur le don est inédite. Nous revenons sur les limites de la contrainte et de l'échange qui justifient pour Perroux le recours au don pour mieux comprendre les réalités économiques. L'originalité de Perroux est de lier don et œuvre commune. La participation se manifeste dans l'organisation par la volonté de ses participants de donner et de se donner à l'œuvre commune. Les travaux qui appellent à une reconnaissance du don dans les organisations et l'économie se développent. L'ouvrage de Norbert Alter (2009) sur le don et la coopération en entreprise a stimulé un débat déjà ancien. Il y montrait comment les succès et les échecs collectifs dépendaient des dons des salariés. D'autres travaux académiques étudient des situations aussi variées que les alliances stratégiques, les établissements de santé, le marketing où le don apparait (Gomez et al, 2015 ; Grévin, 2013 ; Masclef, 2012 ; Barel et Fremeaux, 2008, Fremeaux et Michelson, 2013).

Depuis les travaux de Perroux, des travaux ont étudié le lien entre don et participation. Bien qu'ils s'intéressent essentiellement à la composante financière de la participation et qu'ils s'appuient sur le don échange, ces travaux confirment l'intérêt d'analyser la participation à la lumière des théories du don et non de celles de l'échange marchand. Les travaux s'intéressent surtout à la participation 
financière des salariés qui n'est qu'une composante de la participation financière, elle-même n'étant qu'un aspect de la participation. La participation financière recouvre la participation, l'intéressement, l'actionnariat salarié et les options d'achat d'actions lorsque la majorité des salariés en bénéficie. On retrouve ainsi la définition de Shared capitalism proposée par Freeman et al (2010). La participation à la gestion n'a pas fait l'objet à notre connaissance de travaux empiriques mobilisant le don. La participation à la gestion recouvre au moins deux réalités : la participation aux décisions sur le lieu de travail et la participation à la gestion de l'entreprise au travers des organes de gouvernance et des instances représentatives du personnel.

\section{Limites de l'échange et de la contrainte et affirmation de la participation comme expression du don}

Envisager l'économie en dépassant la contrainte et l'échange marchand est le point de vue adopté par François Perroux. Ce dernier considère en effet que les mobiles désintéressés n'ont pas un rendement économique nécessairement plus faible que les mobiles intéressés. Ces mobiles désintéressés peuvent donc être analysés en termes économiques. S'appuyant sur une critique de l'approche économique standard fondée sur l'échange marchand, Perroux propose d'analyser la participation en termes de don. Perroux associe notamment don et participation dans son ouvrage de 1960 intitulé «Economie et société : contrainte, échange, don ». Il conçoit également la participation comme un dépassement de la seule participation aux bénéfices (Perroux, 1970 ; p. 1561). Il ne se borne pas à sa typologie et considère que les trois motifs sont toujours liés.

"Ecartons un faux parallèle contrasté. L'innocent dirait : le plan repose sur la contrainte, le marché sur la liberté. Dans l'ordre jugé couramment "économique »: ici, la trique, et là, la carotte. Le vrai est que, partout, pour obtenir le produit maximum au moindre cô̂t, toutes les motivations sont utilisées. Il est impossible en fait, dans la pratique, de coupler une institution déterminée avec une série déterminée de motivations (...). » (Perroux, $1994 ;$ p. 87)

Il considère en effet que, bien que les sociétés socialistes soient fondées essentiellement sur la contrainte et les sociétés libérales sur l'échange, chacune d'elle peut aussi utiliser respectivement l'échange et la contrainte. Après avoir mis en lumière les limites de l'échange et de la contrainte, il expose sa conception du don. Le don se concrétise pour Perroux dans la participation à l'œuvre commune. Nous avons notamment développé ce lien entre don et participation établi par l'auteur (Aubert, 2013). Pour Caillé et Grésy (2014 ; p. 108-109), les échanges de dons verticaux traduisent le « rapport à l'esprit de l'organisation, à ses valeurs, sa culture, ses objectifs. Le degré d'incorporation de cet esprit par les membres de l'organisation est le déterminant ultime de son efficacité. (...) L'organisation demande ainsi à ses membres de participer à une œuvre commune ». Perroux développe très tôt sa conception de la communauté ou de l'œuvre commune dans un ouvrage intitulé «Communauté » (Perroux, 1942). A cette époque, il parle plutôt d'adhésion des consciences que de don mais reconnait que c'est l'association des trois éléments de la contrainte, de l'échange et du don qui contribue à l'accomplissement d'une œuvre commune constitutive de la communauté (Perroux, 1942 ; p. 43 et p. 62). Dans les travaux de Perroux, on retrouve le triptyque contrainte échange don qui 
trouve sa finalisation dans l'ouvrage de 1960. Perroux (1942) relève que la diversité des formes communautaires peut être analysée comme «l'œuvre de la contrainte, de l'échange de services et de l'interaction psychique» (p. 39). Le parallèle avec les trois motifs est évident et l'économiste développe en précisant que l'intégration dans la communauté peut se produire : - par élimination des individus qui n'acceptent pas les buts du groupement ou en contrarient la réalisation; - par transactions pour parvenir à un compromis entre les individus qui ont des intérêts opposés ; - par la synthèse dans le cas où « des individus opposés au sein d'un groupement découvrent un objet commun au-delà de ce qui les sépare » (p. 40). Il se positionne nettement en faveur de la synthèse « qui propose aux classes antagonistes des objets communs. Elle exprime et valorise ce que, malgré toutes leurs déclarations, elles ont été obligées de poursuivre (prospérité de l'entreprise et de la profession) » (p. 42). La synthèse fait des règles juridiques et sociales des moyens d'expression d'objets communs et de valeurs communes. Perroux (1947) évoque encore un autre triptyque qui recoupe à nouveau celui de la contrainte, de l'échange et du don : les actes conditionnés, les actes calculés, les actes inspirés. Selon Villey et Nême (1996), cette typologie est évoquée par Perroux pour contester l'idée que l'ordre de l'infra-rationnel (activité instinctive ou réflexe) ou du supra-rationnel (activité esthétique ou mystique) échapperaient à toute activité économique.

A la lumière des développements récents sur le don, des dysfonctionnements au sein des organisations relèveraient de l'absence de reconnaissance du don. On reconnait ainsi l'importance de l'analyse de Perroux si l'on retient que l'un des trois facteurs du bonheur au travail avec la passion pour son métier et le sentiment de reconnaissance (Enquête Vivavoice pour le Nouvel Observateur, 24 octobre 2013) est selon Yves Clot le sentiment « de participer à une œuvre collective et concrète, une construction qui dure et que l'on peut montrer » (Clot, 2010 cité par Caillé et Grésy, 2014).

\subsection{Limites de l'échange et de la contrainte et affirmation du don comme motif de l'acte économique}

Perroux associe à chacune des doctrines libérale et marxiste une motivation de l'acte économique ${ }^{5}$. La contrainte est fondée sur l'ordre total des préférences, l'échange sur l'équivalence et le don sur la participation. Chez les marxistes, la motivation de l'acte économique est la contrainte. En revanche, pour les libéraux, la motivation de l'acte économique est l'échange. Sa vision des relations économiques comme la coïncidence de conflits et de coopérations (lutte/concours) l'empêche de se rattacher exclusivement à l'une de ces deux doctrines. Il identifie une troisième motivation de l'acte économique qui semble apparaître comme un compromis entre échange et contrainte : le don.

"L'économie intelligible, dans une société donnée, résulte de la combinaison de trois flux, chacun d'eux ne pouvant être compris que par référence aux deux autres : le flux des opérations marchandes, le flux des aides (transferts de solidarité) et le flux des prélèvements contraints. » (Perroux, 1990 ; p. 541)

\footnotetext{
${ }^{5}$ L'acte économique est « la lutte ou le concours (typiquement la lutte-concours) par quoi un individu acquiert et emploie des choses comptabilisables » (Perroux, 1994 ; p. 77).
} 
Les limites des théories libérale et marxiste sont d'ordre conceptuel et méthodologique. Après une démonstration mobilisant notamment les travaux de Kenneth Arrow, Perroux conclue «qu'il n'est donc pas plus possible, jusqu'ici, en termes rationnels et analytiques, de construire le choix social coïncidant avec les choix individuels, qu'il ne l'a été de construire l'équivalence sociale et l'équivalence entre individus à partir des théories courantes du marché »(Perroux ; 1994, p. 115).

Il met ainsi en lumière les limites des raisonnements fondés sur l'équivalence - soit l'échange marchand de la théorie libérale - et sur la contrainte - soit l'ordre total des préférences de la théorie marxiste. Il accuse donc le raisonnement marginaliste de s'être débarrassé des considérations morales de ses précurseurs. A ce titre, il rappelle le contenu d'un texte d'Alfred Marshall sur la chevalerie économique et les développements de John Stuart Mill sur l'état stationnaire. "La chevalerie dans l'exercice de la vie économique va de pair avec la chevalerie dans l'usage de la richesse : son code est observé par l'homme riche qui donne à son peuple ce qu'il a reçu. Entériné par l'opinion publique, ce code ne régirait pas seulement tels individus, ni même l'individu, mais l'entière communauté » (Marshall, 1907; p. 27 cité par Perroux, 1954 ; p. 20). Pour John Stuart Mill, « le meilleur état, pour la nature humaine, est celui où personne n'est pauvre, où personne ne désire devenir plus riche, où nul n'a de raison de croire qu'il sera renversé dans la mêlée avec d'autres individus qui se jettent en avant » (Mill, 1929 ; p. 749 cité par Perroux, 1994 ; p. 33). On comprend l'intérêt de Perroux pour les réflexions de ces auteurs en les trouvant mentionnées et développées dans deux de ses travaux de 1954 et de 1960. Il trouve ainsi la justification de son propos sur les limites de l'échange marchand dans les réflexions de ces deux auteurs majeurs de la pensée économique libérale du $19^{\text {ème }}$ siècle.

La notion d'équivalence qui est essentielle à l'échange marchand oriente l'attention vers les choses comptabilisables. C'est une autre limite de l'analyse libérale selon Perroux, d'ordre méthodologique dans ce cas. Cette tendance à centrer l'analyse sur les choses comptabilisables conduit les libéraux à proposer une explication partielle de la réalité observable. Cet écueil est lui-même la conséquence du choix conceptuel des libéraux déjà évoqué : l'accent mis sur l'échange marchand. La conséquence de ce choix est la focalisation sur les réalités qui trouvent une expression monétaire. Ce faisant, ils excluent trois faits : la spécificité des mobiles désintéressés, l'échec des calculs individuels et marginaux, les représentations des groupes (Perroux, 1954 ; p. 16).

On retrouve parmi les trois faits mentionnés par Perroux des intuitions qui seront ensuite développées par les économistes. L'échec des calculs individuels et marginaux trouvera par exemple sa conceptualisation dans les travaux d'Herbert Simon et, plus tard, de Kahneman et Tversky. Nous nous intéressons ici à la première réalité mentionnée par Perroux et ignorée par les néoclassiques : la spécificité des mobiles désintéressés. Pour l'économiste, l'analyse marginaliste exclue une partie des mobiles humains, pire, elle exclurait l'essentiel des mobiles humains : «On a pu croire que les gens travaillent pour gagner: le certain est qu'ils ne travaillent pas que pour gagner et, peut-être, pas principalement pour gagner. » (Perroux, 1994 ; p. 91). C'est ici une des principales découverte de la 
science économique contemporaine qu'anticipe l'auteur et mis en lumière par Frey (1997) : la distinction entre motivations intrinsèques et extrinsèques.

Perroux considère que le don, qui repose sur la participation, permet la mise en œuvre et en valeur de la ressource humaine. En effet, l'auteur indique que la mise en valeur de la ressource humaine est indissociable du don des agents par leur participation à une œuvre commune. Pour démontrer cet argument, nous rappelons d'abord que Perroux considère que les échanges marchands sont certes des échanges d'objets mais avant tout des échanges entre agents auxquels l'économie doit s'intéresser en priorité.

\subsection{La participation comme expression du don}

Perroux retient trois motifs de l'acte économique : la contrainte, l'échange et le don. L'originalité de la pensée de Perroux réside donc dans l'attention portée au troisième motif. Le don repose sur la participation.

«D'une part l'échange, la contrainte et le don ont chacun sa logique propre. L'échange est à base d'équivalence et de réciprocité. La contrainte a pour fondement un ordre total des préférences de tous les agents qui composent la société globale. Le don - pseudo-don ou don véritable - repose sur la participation; cette dernière se présente sous des formes extérieures et limitées dans les transferts de solidarité, ou sous des modes profonds et intimes dans les dons d'amour. »(Perroux, $1994 ;$; p. 94)

La prise en compte du don permet de dépasser les analyses fondées sur l'échange marchand dont Perroux souligne les limites car les aspirations humaines ne peuvent se résumer à la recherche du maximum de confort (Perroux, 1970 ; p. 1562). C'est dans l'acte gratuit que l'on peut trouver une réponse aux aspirations profondes. Mais l'acte gratuit n'est cependant pas dénué de toute rationalité. L'acte gratuit serait même au contraire empreint d'une rationalité proprement humaine (Perroux, 1994 ; p. 84). Perroux considère que la théorie économique contemporaine exclut les aspirations humaines au don au motif qu'elles ne seraient pas économiques. D'autres disciplines reconnaissent pourtant l'intérêt d'analyser ces motifs (Perroux, 1994 ; p. 82). Au-delà de leur prétendu non économicité, les motivations écartées par la théorie économique seraient donc aussi rejetées au motif qu'elles ne seraient pas égocentrées. Pourtant, lors d'un échange marchand, ce sont davantage que des biens de valeurs équivalentes qui sont échangés. Car l'échange marchand s'accompagne en réalité de relations qui n'ont pas d'expression monétaire. La socialité de l'échange marchand est incluse dans une socialité plus vaste où le gratuit serait la règle et l'onéreux l'exception.

«De plus, l'échange économique est soigneusement extrait et supposé isolable de ses accompagnements sociaux. Or, dans une société organisée, les hommes ne peuvent pas échanger uniquement et exclusivement une marchandise. Ils échangent, à cette occasion, des symboles, des significations, des services et des informations. Chaque marchandise devrait être considérée comme le noyau de services non imputables qui la qualifient socialement, et qui - bénéfiques ou nuisibles - sont gratuits en ce sens élémentaires qu'ils ne sont pas payés. Si la société marchande les exclut, c'est pour simplifier et justifier ses comptes. » (Perroux, $1994 ;$ p. 13)

La socialité de l'engagement dépasserait donc selon Perroux la socialité plus étroite de l'échange marchand. Les échanges qui donnent naissance à la socialité de l'engagement ne sont pas assujettis à 
l'équivalence. Or, l'économiste explique que la notion d'équivalence qui fonde l'échange marchand comporte des limites et est toujours difficile à obtenir.

"Pour signaler le passage sur un plan nouveau qu'entraîne la socialité de l'engagement, il n'est que d'interroger les habitudes de langage des Occidentaux. On "se dévoue " à l'Etat (on se dévouerait éventuellement à un plan qui désigne une cuvre collective), on ne "se dévoue » pas au marché. On donne sa foi à une personne ou même à un parti, on ne la donne pas à un quasi mécanisme anonyme. Se dévouer, donner sa foi, demander réciproquement le dévouement et la foi d'un autre, c'est entrer dans un échange où l'équivalence est impossible. L'engagement va au-delà du contrat et de l'association, de l'Etat et de la société (note de l'auteur : "à base d'imperium »). » (Perroux, 1994 ; p. 104)

Alors pourquoi la théorie économique étudie exclusivement l'échange marchand ? Ce n'est que parce que les grandeurs monétaires se prêtent plus aisément au calcul économique que l'échange onéreux devient la règle de la théorie économique. Les autres formes d'interactions sont sous-estimées voire gommées.

"Pseudo-dons et dons ne rentrent pas dans les boîtes que la théorie économique a fabriquées. Pour
qu'une opération rentre dans le jeu de ces boîtes, il faut d'abord qu'elle rentre dans la boîte
élémentaire: l'échange onéreux. (..) De part et d'autre, l'échange est individuel, libre, onéreux,
calculable et calculé. Voilà qui est entièrement étanche aux pseudo-dons et aux dons intervenant entre
des groupes sociaux, des nations. » (Perroux, $1954 ; p .6)$

En se focalisant sur l'échange marchand mesurable, l'économie est incapable d'analyser la ressource humaine, notion essentielle pour Perroux qu'il distingue nettement du capital humain ${ }^{6}$ et qui est source de prospérité. Afin de cerner le concept de ressource humaine, il faut donc dépasser les analyses reposant exclusivement sur l'échange et intégrer la complexité des interactions économiques, c'est-àdire les logiques entremêlées de la contrainte, de l'échange et du don.

Après avoir insisté sur la nécessité de dépasser les analyses centrées sur l'échange marchand et la contrainte, abordons maintenant la conception du don de Perroux. Il écarte tout d'abord les interrogations sur l'existence de dons purs qui consistent à s'intéresser à l'existence de dons totalement désintéressés. Pour l'auteur, que les dons purs existent ou pas, ils n'en animent pas moins les consciences et les actions humaines. L'idée du don pur surpasse ainsi sa réalité.

"Philosophies, religions, littératures l'attestent : quand bien même il n'y aurait jamais eu, sous les cieux, un seul acte de don parfaitement pur, on pourrait encore tenir pour certain que les hommes en ont formé et vénéré l'idée et que cette idée a retenti dans les consciences individuelles et dans les représentations des groupes. »(Perroux, $1954 ;$ p. 18).

La participation est en revanche un fait observable : celui du don à l'œuvre commune. Le don à l'œuvre commune marque le passage de l'individuel au collectif et lie ainsi don et participation. En réalité, toute participation à des œuvres communes donne lieu au don de soi. L'autre monde, la Patrie, la Cité, le Parti, la Cause, l'entreprise sont cités en exemple.

La participation est «une finalité de toutes les sociétés organisées» (Perroux, 1970; p. 1561). Elle découle avant tout de sa critique de l'échange et de la contrainte et se décline différemment au niveau macroéconomique ou microéconomique. Il appelle de ses vœux une société de participation répondant aux aspirations de la masse. Il critique également la mise en œuvre partielle de la participation dans les entreprises capitalistes par la seule participation aux bénéfices. Les entreprises capitalistes sont pour

\footnotetext{
${ }^{6}$ «Cette constatation et cet énoncé dissipent l'illusion de l'économisme incluse dans le pseudo-concept de « capital humain » et dans les mesures numériques qu'on s'efforce d'en prendre » Perroux (1990; p. 540).
} 
lui les lieux d'un conflit permanent entre des intérêts divergents, ceux du travail et ceux du capital. Ce conflit résulte de l'opposition entre les idéologies inspirées de l'échange et de la contrainte. Les conséquences de ce conflit au sein des entreprises sont supportées par l'ensemble du corps social.

"Cependant, l'entreprise résiste, car elle est le centre producteur, l'unité économique du système capitaliste. Maintenir juridiquement les choses en l'état actuel, c'est accepter une lutte dont le pays fait les frais, aussi le Gouvernement Provisoire de la République Française a-t-il promulgué une ordonnance instituant "les comités d'entreprise", amorce d'une participation plus étendue des salariés aux responsabilités jusqu'ici assumée par les seuls entrepreneurs. »(Perroux, 1947 ; p. 1)

Par l'institution des comités d'entreprise, c'est la participation des salariés aux responsabilités de l'entrepreneur que le gouvernement provisoire de la République française entendait favoriser. Ses propos sont sans équivoque, Perroux juge que salariés et entrepreneurs sont appelés à "gérer en commun des ensembles industriels » (Perroux, 1947 ; p. 1]. La participation aux bénéfices deviendrait un alibi en réduisant la participation à une simple rationalité économique la vidant de son sens. La participation aux bénéfices se limite en effet au partage des profits et du capital en occultant des caractéristiques essentielles de la participation. La participation à l'œuvre commune est ainsi à nouveau placée au premier plan. En ce qui concerne le partage des profits, qui en décide et comment est la question essentielle pour l'économiste. Elle renvoie à un concept fondamental des travaux de l'auteur : le pouvoir.

«Dépendants comme nous le sommes des structures marchandes, la participation économique nous paraît tenir tout entière et s'épuiser dans la rationalité économique telle que nous l'offrent, simplette, appauvrie, anémique, les analyses reçues. Les agents et leur groupe participent à l'effort; le chef d'entreprise fait son métier et le travailleur tente de participer à la gestion de l'entreprise. Lui et eux participent aux fruits : au bénéfice et au capital. Qui fait les parts et comment? L'indiscrète question est éloignée, par convention, des discussions économiques. En paraissent exclues aussi les participations aux communautés de vie et de destin, et aux communautés des plus hautes valeurs, immanentes ou transcendantes »(Perroux, $1994 ;$ p. 123).

Les entrepreneurs et les ouvriers utiliseraient l'alibi de la participation aux bénéfices justement du fait de leur subordination aux idéologies inspirées de la contrainte et de l'échange. Les syndicats ouvriers refusaient de se compromettre en participant à la gestion des entreprises et les entrepreneurs refusaient la participation ouvrière (Perroux, 1947 ; p. 1).

Perroux considérait néanmoins que les intérêts économiques et les convictions idéologiques seraient dépassés pour aboutir à une forme de codétermination. Il aurait résulté des évolutions qu'il anticipait un renversement faisant de la participation des salariés à la gestion des entreprises une cause de la participation économique et non une conséquence attendue des gaullistes. C'est en effet sans doute en envisageant que la participation à la gestion pouvait être une cause de la participation aux bénéfices et au capital que le législateur a envisagé les dispositifs d'épargne salariale. On peut reprocher à Perroux son corporatisme qu'il envisage comme une troisième voie. Delsol (2015) note que Perroux (1938) différenciait lui-même le corporatisme stricto sensu du corporatisme lato sensu. C'est seulement dans le second que des groupes autonomes gèrent librement leur production et leur vie sociale. Le premier fondait selon lui «des groupes non autonomes, mais publics ou semi publics, organisant l'économie de façon autoritaire $($ Delsol, 2015 ; p. 248). Il préconisait l'intervention de l'Etat pour éviter les dérives totalitaires du corporatisme (Perroux, 1938 ; p. 154 cité par Delsol, 2015). Le corporatisme de 
Perroux s'exprime dans la communauté de travail réunissant en nombre égal des représentants du capital et du travail et où l'Etat intervient comme un arbitre des conflits en dernier ressort (Perroux, 1938 ; p. 197 cité par Cohen, 2006).

\section{Les travaux sur la participation comme expression du don}

Un parallèle est ainsi établi entre la contrainte, l'échange et le don d'une part, l'ordre total des préférences, l'équivalence et la participation d'autre part. L'analyse paraît datée car elle fait référence au $20^{\text {ème }}$ siècle marqué par l'opposition idéologique entre libéralisme et marxisme. Actualiser le schéma perrouxien de la contrainte, de l'échange et du don est possible en le transposant à celui de la bureaucratisation, de la libéra(lisa)tion et de la participation. La bureaucratisation pour désigner les contraintes auxquelles sont soumises les organisations publiques et privées et dont on aurait bien tort de considérer qu'elle est du seul fait des organisations publiques. Comme l'indique Graeber (2015), «il faut mille fois plus de paperasse pour entretenir une économie de marché libre que la monarchie absolue de Louis XIV » (couverture de l'ouvrage). Cette «cage d'acier» de la bureaucratie consiste en un système complexe de contrôle des comportements qui font des organisations des panoptiques inversés selon Gomez (2013) au travers desquelles le marché doit tout voir. Detchessahar (2015) considère que la place prise par les marchés et la très grande entreprise donne aux personnes le sentiment d'être contrôlées par des forces extérieures. Toujours d'après Detchessahar (2015), non seulement la technicité des prescriptions objectives (procédures, standards, process) et subjectives (chartes, valeurs, script...) du management sont nombreuses et complexes mais cette technicité éloigne du débat les personnes qu'elle concerne pourtant mais qui ne les comprennent plus. Le travail objectivé, mesuré est ainsi débarrassé de toute dimension morale. Les remèdes du management deviennent le mal pour des organisations «malades de gestionnite » (Detchessahar et Grévin, 2009) où les managers passent plus de temps à évaluer le travail qu'à travailler. L'évaluation est ainsi la nouvelle contrainte qui pèse sur les organisations et leurs membres. La libération des entreprises répond à cette pression bureaucratique. Nous faisons référence aux débats suscités par l'entreprise libérée, notamment après la parution du bestseller «Freedom inc. » (Carney et Getz, 2009) et de sa traduction française «Liberté et cie » (Getz et Carney, 2012). Si elle est présentée sous son meilleur jour par ses promoteurs, la forme la plus aboutie de la libération n'est-elle pas la libéralisation ? Elle ferait étrangement penser au nœud de contrats des économistes du renouveau libéral des années 1970, les mêmes tenants des théories contractuelles de l'organisation précédemment cités. Selon cette vision, l'entreprise n'est plus une communauté mais une somme de relations interindividuelles entre individus autonomes matérialisées par des contrats. L'entreprise n'y existe plus en tant que telle que comme une fiction juridique. La libération desserre le carcan bureaucratique et hiérarchique, la libéralisation coupe les salariés de tout lien justifiant ainsi, à l'extrême, la fin du salariat et des liens de subordination. Les nouvelles formes d'organisation du travail regroupent des pratiques aussi diverses que le travail en équipes autonomes, le travail à distance et les contrats zéro heure. L'actualité de l'analyse de Perroux 
en termes de contrainte, échange et don est plus évidente à l'aune des phénomènes actuels de bureaucratisation et de libéralisation dont nous accentuons ici les contrastes à dessein. Car l'originalité de Perroux est de ne pas séparer les motifs en établissant une typologie étanche. Entre les deux mouvements présents dans l'actualité des entreprises, le don est moins visible. Avec Perroux, il se donne à voir par les pratiques participatives. Or, la recherche s'est pour l'instant concentrée sur la participation aux bénéfices des salariés. Perroux et ses contemporains gaullistes considéraient qu'elle ne résumait pas la participation. La participation aux bénéfices est une composante de la participation mais elle est aussi une préconisation majeure des théories contractuelles de l'organisation. Dans l'esprit de ces approches, pallier les conséquences des penchants égoïstes de l'homo æeconomicus suppose d'aligner les intérêts financiers. C'est ainsi que les travaux liant don et participation se sont surtout inscrits dans ce courant dominant en se focalisant sur la participation aux bénéfices.

\subsection{Don, participation et réciprocité}

La participation des salariés peut prendre plusieurs formes : la participation aux décisions sur le lieu de travail ; la participation aux bénéfices ; la participation à la gestion. Les critiques de la participation aux bénéfices concernent surtout l'inefficience des incitations collectives, le contrôle des organisations participatives et l'exposition au risque excessive qui résulteraient de la participation au capital des entreprises. L'inefficience des incitations collectives a été la plus documentée par la théorie économique. Nous l'évoquons à nouveau mais dans le but de mettre en évidence l'importance du don qui ont d'ores-et-déjà apporté des réponses à ces critiques. Si l'on admet l'hypothèse de la complémentarité des formes de participation, les questions du contrôle et de l'exposition au risque, bien qu'ayant pas fait l'objet d'autant de travaux, peuvent également être appréhendées grâce au don. Les incitations collectives (participation, intéressement et actionnariat salarié) se heurtent en effet à un problème de taille : les comportements de passager clandestin. Des incitations collectives consistant à accorder à chacun la même part du profit généré tendent inexorablement à décourager les efforts individus, chacun cherchant à profiter du travail des autres (Holmström, 1982). Comme le célèbre article d'Alchian et Demsetz (1972) le suggère, il est également couteux voire impossible d'évaluer les efforts que chaque salarié apportent à la réalisation d'une tâche collective. Le contrôle tend à être assuré par le créancier résiduel. Les salariés eux, reçoivent un salaire qui reflète leur niveau de productivité et ne sont pas intéressés aux profits de l'entreprise. Leur conclusion légitime la structure de rémunérations des entreprises capitalistes.

Putterman (2006) indique pourtant que plusieurs modèles théoriques formalisés ne parviennent pas forcément à ces conclusions. Putterman (2006) montre que les premières tentatives de modélisations des incitations collectives pratiquées dans les fermes collectives (chinoises, soviétiques ou israéliennes) montraient leur inefficience mais en raison d'un surcroit de travail. Ces modèles n'intégraient pas d'interdépendance entre les agents qui pouvaient se traduire notamment par leur altruisme. Ce fut chose faite avec Amartya Sen (1966) qui intégre un paramètre de sympathie dans son 
modèle. Techniquement, cela consiste à considérer qu'une proportion de l'utilité d'un agent dépend de celle d'un autre agent. Lorsque ce paramètre de sympathie est très faible, la distribution égalitaire des profits décourage l'effort. Mais si l'agent valorise autant l'utilité des autres que la sienne, la distribution égalitaire des revenus est efficiente. Le modèle de Sen montre néanmoins que toute interdépendance limite les effets des comportements de passager clandestin.

Une autre forme d'interdépendance a été prise en compte par les économistes et les sociologues : la réciprocité. Kolm (2006, p. 25, notre traduction) définit la réciprocité :

"Un don ou une faveur motivé par un autre don, par exemple le retour d'un don motivé par un autre don, constitue l'importante relation sociale de la réciprocité. Elle est très différente d'un échange intéressé où chaque transfert (ou faveur) est réalisé à la condition qu'un autre transfert soit effectué, et n'est donc pas un don (au sens propre du terme). »

Kolm décrit trois motifs de la réciprocité qui peuvent être combinés : la réciprocité équilibrée (balance reciprocity), la réciprocité affective (liking reciprocity), la réciprocité de continuation (continuation reciprocity). La première maintient l'équilibre des dons en évitant tout endettement moral, la seconde intervient du fait d'un lien affectif entre donneur et receveur, la troisième a pour objectif de poursuivre une relation en induisant un nouveau don. On considère ici uniquement la réciprocité positive qui fait intervenir des dons et non la douleur ou le mal (réciprocité négative qui implique que l'on rend le mal). Cette réciprocité négative n'est pourtant pas inutile pour comprendre la participation. Imaginons simplement un actionnaire salarié qui aurait perdu son épargne en l'investissant dans son entreprise (comme ce fut le cas pour le célèbre cas d'Enron). On peut imaginer que cette perte puisse l'inciter à adopter un comportement négatif vis-à-vis de son employeur. On constate que la réciprocité est particulièrement développée au sein des groupes qui ont de multiples interactions. Le lieu de travail est donc un lieu privilégié d'observation de la réciprocité. D'après Putterman (2006), la réciprocité ne peut pas être appréhendée de façon strictement rationnelle mais plutôt comme une inclinaison qui est dû à l'évolution (influence des expériences passées qui fondent la réputation), à la socialisation et aux pressions sociales. Les comportements réciprocitaires seraient non seulement la conséquence du désir des individus d'être perçus comme de bonnes personnes mais également de se considérer elles-mêmes comme de bonnes personnes. La rationalité économique néo-classique ne serait pas en mesure de comprendre ces comportements, pourtant au cœur de la coopération sur le lieu de travail. La réciprocité n'est cependant pas dépourvue de calcul économique. On retrouve dans ces deux dernières affirmations - inaptitude de l'échange marchand à appréhender la logique du don et présence du calcul économique dans cette logique - les intitulions de Perroux. Pour analyser la participation, il faudrait donc davantage se situer dans la logique du don-échange (gift exchange) que celle du don-gratuit (giftgiving) que Masclef (2013) distingue.

La conception du don-échange que l'on retrouve dans la réciprocité fait référence aux travaux de Mauss (1950). Cette conception s'applique aux relations entre employeurs et employés lorsque l'on analyse la participation en particulier et les relations d'emploi en général. La traduction anglaise du don échange (gift exchange) renvoie aux travaux d'Akerlof (1982). Akerlof (1982) s'intéresse à une 
étude montrant que dans une entreprise, les salariés travaillent davantage que les quotas requis et certains plus que d'autres. Cette situation est impossible du point de vue de la théorie néo-classique. Soit les salariés les plus performants devraient diminuer leur productivité, soit l'entreprise devrait aligner les normes de travail sur celles des plus productifs. L'explication de ce phénomène par Akerlof s'appuie explicitement sur Mauss (1950). L'effort plus élevé que la normale peut s'analyser en termes de don. Ces derniers attendent un contre don de leur employeur qui peut prendre plusieurs formes : un salaire plus élevé que le salaire de marché, de meilleures conditions de travail et pas de différences de salaire entre les membres du groupe.

"Du point de vue du travailleur, le "don" donné est le travail au-delà du minimum requis; et du point de vue de l'entreprise le "don" donné est le salaire en plus de ce que ces femmes pourraient recevoir si elles quittaient leurs emplois actuels. La conséquence des liens qui unissent les travailleurs est que l'entreprise ne peut pas contracter avec chaque salarié individuellement, mais doit traiter l'ensemble du groupe de salariés avec les mêmes normes, collectivement. » (Akerlof, 1982; p. 544, notre traduction)

Comme dans le cas d'un don émanant d'un salarié, l'employeur attend en retour un contre don de ses salariés. Comme avec Perroux, la référence à Mauss est explicite et le don échange paraît privilégié. Akerlof laisse cependant entrevoir une conception qui n'est pas strictement limitée au don échange. Du point de vue macroéconomique, ces comportements peuvent aboutir à un salaire plus élevé que le salaire d'équilibre résultant en un chômage involontaire. En effet, l'entreprise peut refuser d'embaucher un demandeur d'emploi pour ne pas remettre en cause les échanges de dons avec le reste de ses salariés qui aboutirait à une chute de leur productivité. Putterman (2006, p. 1422) présente la littérature en économie expérimentale qui teste les prédictions de l'économie du don. La situation reproduite en laboratoire et qui traduit assez fidèlement les caractéristiques du lieu de travail avec participation financière est le mécanisme de contribution volontaire (voluntary contribution mechanism, Isaac et Walker (1988a, 1988b). L'expérience consiste à attribuer à chaque agent une somme d'argent au début d'un jeu. Les participants doivent décider de la proportion de cette somme qui sera allouée à leur compte individuel, la proportion restante étant allouée au groupe. Sur le lieu de travail, la première stratégie s'apparente à un comportement de passager clandestin alors que la seconde correspondrait à un comportement de travail coopératif. La proportion allouée au groupe est multipliée par un facteur d'échelle qui représente les économies d'échelle résultant du travail coopératif. Le total de la somme allouée par les membres au groupe est augmenté par le facteur d'échelle et partagée en parts égales entre les membres du groupe. A titre d'exemple, si la dotation initiale de chacun des membres d'un groupe de quatre est de $10 \$$, que le facteur d'échelle est de 2, et que tous choisissent d'allouer la totalité de leur dotation au groupe soit $40 \$$, alors chacun reçoit $20 \$$ $([40 \$ \times 2] / 4)$. Si aucun des membres du groupe ne participe à la dotation du groupe, alors chaque membre reçoit $10 \$$. En revanche, si trois des membres participent pour $10 \$$ et que le dernier n'apporte rien, les trois premiers reçoivent $15([3 \times 10 \$ \times 2] / 4)$ alors que le passager clandestin reçoit 25 $(10 \$+15 \$)$. Cette situation traduit bien l'incitation de chacun des membres d'un groupe de travail à adopter une stratégie de passager clandestin en présence d'incitations collectives. Dans cette configuration qui correspond à un dilemme de prisonnier, la théorie économique standard prédit que 
tous les membres adoptent un comportement de passager clandestin. Les expériences menées ont repris ce schéma en faisant varier ses caractéristiques. On constate que les participants allouent en moyenne $60 \%$ de leur dotation mais que cette dotation diminue lorsque le jeu est répété. Parmi les variantes de ce jeu qui contredisent cette baisse du niveau des contributions, on peut citer plusieurs résultats. Les contributions demeurent élevées si : - le jeu est arrêté et repris depuis le début ; - des groupes sont formés de participants qui se sont révélés être des contributeurs élevés ; - les participants peuvent communiquer avant de jouer; - les participants peuvent punir les autres participants de n'avoir pas participé (réciprocité négative). Avec Kreps et al (1982), on peut également comprendre les comportements de dons à la lumière des croyances et des préférences des agents sur la composition du groupe auxquels ils appartiennent. En effet, un agent généreux convaincu que les membres de son groupe partagent ses préférences contribuera vraisemblablement davantage. En ce qui concerne la réciprocité négative, elle peut dissuader les comportements de passagers clandestins. Les expériences décrites par Putterman (2006) indiquent la présence de tels comportements. Les contributeurs élevés (positive reciprocators) peuvent en effet punir les passagers clandestins au-delà de leur propre intérêt et même punir les comportements de passager clandestin lorsque ces derniers profitent d'un tiers. La réciprocité négative inciterait ainsi les passagers clandestins à se comporter comme des contributeurs élevés. On retrouve un argument important en faveur des systèmes d'incitation collectifs : le contrôle horizontal. Selon Kandel et Lazear (1992), le contrôle horizontal peut limiter les comportements de passager clandestin à condition notamment qu'un système d'incitation collectif soit mis en œuvre, que les membres du groupe réalisent des tâches similaires et que lorsque la taille du groupe n'est pas trop importante. Sur le lieu de travail, les sanctions se traduisent par des réprimandes verbales ou des interactions moins nombreuses avec les passagers clandestins. Au titre de la réciprocité négative, il faut enfin mentionner que les plus coopératifs peuvent aussi faire l'objet de sanctions de la part des passagers clandestins. Ces derniers peuvent être motivés par le désir de vengeance contre ceux qui les ont sanctionnés auparavant, le rejet du comportement moral des plus coopératifs ou le souhait de diminuer leurs revenus. Réciprocité positive et négative sont donc à l'œuvre dans les relations de travail impliquant la participation financière.

\subsection{Les travaux empiriques sur la participation comme expression du don}

Malgré les nombreux travaux empiriques sur les relations entre la participation des salariés et la performance des entreprises, il n'existe pas à notre connaissance de théorie permettant de les expliquer. Les courants théoriques les plus souvent cités sont l'asymétrie d'information, la théorie de l'agence et le modèle principal agent. Leurs fondements tentent d'expliquer les relations entre salariés et dirigeants en se fondant sur l'échange marchand. Perroux considérait que le don expliquait mieux la participation et que cette relation entre don et participation est de nature à fonder une théorie de la participation (Aubert, 2013). Une telle théorie permettrait de mieux appréhender les effets de la participation sur la performance de l'entreprise. En effet, la littérature empirique soutient la thèse de la 
complémentarité des formes de participation. Ce faisant, elle suggère d'appréhender la participation comme un tout et non comme un ensemble de pratiques distinctes. Contrairement à ce que propose l'approche néo-classique, la participation ne se résumerait pas à l'échange marchand c'est-à-dire à la participation financière des salariés. L'intuition de Perroux a récemment coïncidé avec plusieurs travaux empiriques. Freeman et al (2010) ont tout d'abord proposé un travail qui est sans doute le plus importante jamais entrepris sur la participation des salariés. Les auteurs ont en effet collecté des données auprès d'un échantillon représentatif de près de 40000 salariés dans plusieurs centaines d'entreprises américaines et britanniques dans la cadre du National Bureau of Economic Research (désormais NBER) Shared Capitalism project. Outre les nombreuses contributions empiriques qui font de cet ouvrage une entreprise inégalée à ce jour, les auteurs inaugurent le concept de Shared capitalism qu'ils définissent sommairement de la façon suivante: "des relations d'emploi où la rémunération ou le patrimoine des travailleurs est directement lié à la performance du lieu de travail ou de l'entreprise» (p. 1, notre traduction). Ils complètent cette définition plus loin: "Cette participation peut prendre plusieurs formes, dont la participation aux bénéfices, l'intéressement, les bonus, l'actionnariat salarié et les stock-options pour l'ensemble des salariés. Toutes ces pratiques ont une chose en commun: offrir au travailleur une part des profits ou de la plus-value de l'action quand l'entreprise fait des bénéfices. Nous appelons l'ensemble de ces pratiques "shared capitalism" »(p. 41, notre traduction). La définition du shared capitalism peut paraître décevante aux premiers abords car restreinte à la participation financière mais il faut la resituer dans le contexte nordaméricain. Il est en revanche intéressant de constater que les résultats de Freeman et al (2010) indiquent que le shared capitalism exprime tout son potentiel lorsqu'il est associé à la participation des salariés aux décisions à plusieurs niveaux de l'entreprise. Les meilleurs résultats sont obtenus lorsque les salariés font confiance au management de leur entreprise, qu'ils considèrent que les relations de travail sont bonnes, que leur entreprise met en œuvre de bonnes pratiques de management (travail en équipe, formation, sécurité de l'emploi), un faible niveau de supervision et - en écho à Akerlof - que le niveau moyen des rémunérations est égal ou supérieur à celui du marché. D'autres résultats du NBER Shared capitalism project montrent la complémentarité de la participation aux bénéfices et de la participation aux décisions et ses résultats favorables sur la satisfaction des salariés, leur attitude envers leur entreprise et leur intention de départ. A l'issu de leur ouvrage, les auteurs offrent une conclusion très importante pour notre propos et qui peuvent être rapprochées des conclusions expérimentales rappelées plus haut :

\footnotetext{
"Ce livre montre que le shared capitalism surmonte les comportements de passager clandestin et le risque financier pour les salariés [les deux principaux problèmes soulevés par ces formes de rémunération] et que la participation financière qui bénéficie à tous les salariés promeut une culture d'entreprise coopérative y compris dans de grands groupes ou les firmes. Associer le shared capitalism à une culture d'entreprise plus coopérative permet de surmonter le free riding et de générer de meilleures performances. Notre interprétation globale se fonde sur les théories de la réciprocité et de l'échange de dons, ainsi que sur les recherches sur les high performance work practices. De plus en plus de travaux empiriques montrent que la réciprocité joue un rôle important dans les relations économiques et sociales en favorisant des normes de coopération. Des modèles économiques formels montrent également
} 
comment les relations de travail peuvent être comprises à l'aune des théories de la réciprocité et de l'échange de dons. » (Freeman et al, 2010; p. 378, notre traduction)

D'autres travaux relativement récents analysent la participation à l'aune des théories du don. Deux précisions importantes doivent être faites. La première est que c'est essentiellement la conception maussienne du don qui est retenue. Pour les travaux empiriques, référence est toujours faite à Akerlof (1982) qui s'inspirait lui-même et citait l'essai de Marcel Mauss. Le don-échange ou gift exchange prend donc le pas sur le don gratuit. La seconde est que ces travaux se concentrent sur la participation des salariés au capital à travers l'actionnariat salarié. Ces deux spécificités s'appliquent aux travaux empiriques de Bryson et Freeman (2014) et d'Aubert et Hollandts (2015). Cette approche a également été présentée par Andrew Pendleton, auteur britannique de référence sur la participation. Il voit dans le recours au don échange un moyen de comprendre les effets de l'actionnariat salarié sur les attitudes et comportements des salariés, là où le modèle principal agent aurait trouvé ses limites. Il trouve les preuves de cette assertion dans le discours des entreprises et des salariés qui adoptent ou bénéficient de l'actionnariat salarié. Ces termes traduisent d'après lui la présence d'échange de dons. L'attribution d'actions aux salariés initierait des comportements réciprocitaires. En acceptant de devenir actionnaire salarié, les salariés témoigneraient de leur volonté d'entrer dans une relation de réciprocité. Le contre don des salariés serait une plus grande implication au travail, un plus faible absentéisme et un moindre turnover. Pendleton (2011) insiste également sur 1'importance pour les employeurs de signaler qu'elles valorisent la réciprocité en accordant des plans d'actionnariat salarié. Il déduit en revanche que 1'actionnariat salarié peut fonctionner sans être pour autant associé à d'autres pratiques participatives. Le partage d'informations et le souci de protéger le patrimoine des salariés seraient en revanche cruciaux pour maintenir la réciprocité positive. Les travaux empiriques de Bryson et Freeman (2014) et d'Aubert et Hollandts (2015) parviennent à des conclusions similaires confirmant cette approche dans le cadre des augmentations de capital réservées aux salariés (ACRS ou ESPP soit Employee stock purchase plans aux Etats-Unis). Dans le cadre de ces opérations, les salariés se voient proposés d'acquérir des actions de leur entreprise à un prix décoté ( $20 \%$ en France). Ils ont le choix d'acquérir ces actions ou de ne pas participer. Bryson et Freeman (2014) considèrent que les salariés assimilent cette décote à un don de leur employeur. L'approche basée sur l'échange de dons serait validée par les comportements réciprocitaires de ceux qui acceptent de participer. Les auteurs observent en effet que la productivité des participants est plus élevée, que leur taux d'absentéisme et de départ sont plus faibles que les non participants. Au niveau des filiales d'un groupe coté français suivies sur cinq ans, Aubert et Hollandts (2015) parviennent à une conclusion similaire : les filiales dans lesquelles le niveau de la participation aux opérations d'actionnariat est positive sont également celles où le taux d'absentéisme est le plus faible. Cette relation n'est pas confirmée pour le taux de rotation du personnel. A notre connaissance, des travaux similaires n’ont pas été entrepris pour tester la présence de don échange au sein des autres formes de participation. 


\section{Conclusion}

La lecture de Perroux mobilise le don pour comprendre la participation et ses effets pour l'entreprise et ses salariés là où l'échange marchand et la contrainte trouvent leurs limites. La critique de la contrainte et de l'échange traduit les angoisses de son époque sur l'opposition entre les idéologies marxiste et libérale. La mise en œuvre de la participation passe ainsi par la reconnaissance du don dans les organisations et de son articulation avec les autres motifs de l'acte économique. Cette participation est appréhendée comme un don à l'œuvre commune, aux collectifs de travail mis à mal par la bureaucratisation et l'ambivalence du discours sur l'autonomisation des salariés. Dans l'esprit de Perroux et de ses contemporains gaullistes, la participation était un tout. Pas de participation aux bénéfices sans participation aux décisions et inversement. Cette idée est conforme à la thèse de la complémentarité des formes de participation qui produirait les meilleurs effets pour les organisations et leur personnel. Cette idée serait aussi valable au niveau macroéconomique. A la suite de leur ouvrage qui concluait sur l'intérêt du don pour comprendre la participation, Blasi, Kruse et Freeman ont encore élargi leur vision du shared capitalism de l'entreprise à la nation : the citizen's share. Dans une analyse des discours des pères fondateurs des Etats-Unis (Washington, Jefferson, Adams et Madison), ils montrent que ces derniers s'inquiétaient de voir se répandre la démocratie sans que la propriété ne soit elle aussi diffusée au plus grand nombre. Le partage des richesses - à l'époque les terres agricoles - fut la réponse qu'ils apportèrent à cette question (Blasi et al, 2013). Selon les auteurs, cette philosophie alliant démocratie et partage des richesses est un élément déterminant de la réussite économique des Etats-Unis au $20^{\text {ème }}$ siècle. Ils s'inquiètent donc des conséquences de la concentration des richesses - de nos jours le capital des entreprises - dans peu de mains. Participation aux décisions et participation aux bénéfices des salariés nécessitent des dons verticaux (Caillé et Grésy, 2014) des dirigeants vers les salariés. C'est ce que l'on peut retenir par exemple de la lecture de l'ouvrage de Getz et Carney sur les entreprises libérées. Ils y racontent l'histoire de dirigeants charismatiques qui ont choisi de faire confiance à leurs salariés en leur donnant plus d'autonomie. Un don initial qui s'est traduit par l'engagement de leurs salariés et la réussite de leurs entreprises. Kruse et Blasi s'étaient également intéressés à l'histoire de la Silicon Valley en montrant comment ses entreprises avaient réussi en attribuant des stock-options à tous leurs salariés (Blasi et al, 2003). Mais la participation est également horizontale et longitudinale, faite de dons entre travailleurs et de dons entre travailleurs de générations différentes (Caillé et Grésy, 2014).

Mettre en œuvre la participation est toujours d'actualité. En France, elle concrétise les aspirations gaulliennes et demeure une troisième voie entre contrainte et échange marchand: "Il y a une troisième solution, c'est la participation qui, elle, change les conditions de l'homme au milieu de la civilisation moderne »(De Gaulle, Conférence de presse, septembre 1968). 


\section{Références}

Akerlof G. (1982), Labor Contracts as a Partial Gift-exchange, Quarterly Journal of Economics, vol. 97, nº , p. 543-569.

Alchian A. et Demsetz H. (1972), Production, Information Costs, and Economic Organization, American Economic Review, vol. 62, p. 777-795.

Alter, N. (2009), Donner et prendre: la coopération en entreprise, La Découverte.

Aubert N. (2013). Une Théorie de la Participation des Salariés Fondée sur le Don: l'Apport de François Perroux, Economies et Sociétés, vol. 47, n¹, p. 63-92.

Aubert N. et Hollandts X. (2015), How Shared Capitalism Affects Employees' Withdrawal? An Econometric Case Study of a French Listed Company, Journal of Applied Business Research, vol. 13, n³, p. 925-938.

Balligand J.-P. et Foucault J.-B. (2000), L'épargne salariale au cœur du contrat social, La Documentation Française.

Barel Y., et Fremeaux, S. (2008), Le Don Gratuit: le Cas d'un Etablissement Public, Gérer et Comprendre, $\mathrm{n}^{\circ} 4$, p. 80-89.

Bryson A. et Freeman R. (2014), Employee Stock Purchase Plans - Gift or Incentive? Evidence from a Multinational Corporation, IZA Discussion Paper No. 8537, http://ftp.iza.org/dp8537.pdf.

Caillé A. et Grésy J. E. (2014), La révolution du don: le management repensé à la lumière de l'anthropologie, Seuil.

Carney B. M. et Getz, I. (2009), Freedom, Inc. Free your employees and let them lead your business to higher productivity, profits, and growth. New York: Crown Business.

Chatriot A. (2012), La Réforme de l'Entreprise, Vingtième siècle. Revue d'histoire, vol. 2, n¹14, p. 183-197.

Clot Y. (2010), Le travail à cour. Pour en finir avec les risques psychosociaux, La Découverte.

Cohen A. (2006), Du Corporatisme au Keynésianisme, Revue française de science politique, vol. 56, $\mathrm{n}^{\circ} 4$, p. 555-592.

Delsol C. (2015). L'état subsidiaire: Ingérence et non-ingérence de l'Etat - le principe de subsidiarité aux fondements de l'histoire européenne, Lexio, Paris.

Dezes M. (1990), Evolution du courant participationniste, in La politique sociale du général de Gaulle, Actes du colloque de Lille des 8 et 9 décembre 1989, coll. Histoire et littératures régionales, éd. Centre d'histoire de la région du Nord et de l'Europe du nord-ouest.

Detchessahar M. (2015), Le marché n'a pas de morale, Cerf.

Detchessahar M. et Grevin, A. (2009), Un organisme de santé... malade de «gestionnite», Gérer et comprendre, $\mathrm{N}^{\circ} 4$, p. 27-37.

Dezes M. (1990), Evolution du courant participationniste, in La politique sociale du général de Gaulle, Actes du colloque de Lille des 8 et 9 décembre 1989, coll. Histoire et littératures régionales, éd. Centre d'histoire de la région du Nord et de l'Europe du nord-ouest.

Freeman R. Kruse D. et Blasi J. (2010), Shared capitalism at work: employee ownership, profit and gain sharing, and broad-based stock options, NBER, University of Chicago Press.

Frémeaux S. et Michelson G. (2011). 'No Strings Attached': Welcoming the Existential Gift in Business, Journal of Business Ethics, vol. 99, n ${ }^{\circ}$, p. 63-75.

Frey B. S. (1997), Not just for the money, Edward Elgar Publishing.

Getz I. et Carney B. M. (2012), Liberté \& Cie. Quand la liberté des salariés fait le succès des entreprises, trad. française, Paris, Fayard.

Godfrain J. (2000), La Participation, Idée Centrale de la Pensée Gaullienne, Espoir, nº125.

Godfrain J. et Cornut-Gentille F. (2005), Une ambition : la participation pour tous, La Documentation Française.

Gomez P. Y. (2013), Le travail invisible: enquête sur une disparition, Editions F. Bourin.

Gomez P.-Y. Grévin A. et Masclef O. (2015), L'entreprise, une affaire de don : ce que révèlent les sciences de gestion, Nouvelle Cité, collection GRACE.

Graeber D. (2015), Bureaucratie: L'utopie des règles, Éditions Les Liens qui libèrent.

Grévin A. (2013). Pour un Réalisme de la Recherche et du Management: Reconnaître le Don au Cœur du Travail. Economies et Sociétés, vol. 47, n¹, p. 33-62. 
Guiol P. (2009), La participation des salariés à la gestion des entreprises : une lecture historicopolitique, in Auberger M.-N. et Conchon A. (eds), Les administrateurs salariés et la gouvernance d'entreprise, La Documentation Française.

Holmström B. (1982), Moral Hazard in Teams, Bell Journal of Economics, vol. 13, p. 324-340.

Isaac R. M. et Walker, J. (1988a), Group Size Effects in Public Goods Provision: the Voluntary Contribution Mechanism, Quarterly Journal of Economics, vol. 103, n ${ }^{\circ}$, p. 179-199.

Isaac R.M. et Walker J.M. (1988b), Communication and Free-riding Behavior: the Voluntary Contributions Mechanism, Economic Inquiry, vol. 26, p. 585-608.

Kandel E. et E. Lazear (1992), Peer Pressure and Partnerships, Journal of Political Economy, vol. 100, p. 801-817.

Kolm S.-C. (2006), Introduction to the economics of giving, altruism and reciprocity, in Ythier J.-M., Kolm, S.-C., Handbook of the economics of giving, altruism and reciprocity, Palgrave Macmillan.

Kreps D., Milgrom P., Roberts J. et Wilson R. (1982), Rational Cooperation in Finitely Repeated Prisoners' Dilemma, Journal of Economic Theory, vol. 27, p. 245-252.

Loichot M. (1966), La réforme pancapitaliste, Robert Laffont, Paris.

Marshall A. (1907), The Social Possibility of Economic Chivalry, The Economic Journal, vol. 17, p. 7-29.

Masclef O. (2012), Altruisme et Dons Gratuits dans une Dynamique d'Emergence d'Alliance, Revue Française de Gestion, vol. 38, p. 223.

Masclef O. (2013), Le Rôle du Don Gratuit dans l'Entreprise, Economies et Sociétés, série K, vol. 22, pp. 7-31.

Mauss M. (1950), Essai sur le don - forme et raison de l'échange dans les sociétés archaïques, in M. Mauss, Sociologie et Anthropologie, PUF, Coll. Quadrige, pp.145-279.

Mill J. S. (1929), Principles of political economy, Longmans, Green et $\mathrm{C}^{\circ}$, London.

Pendleton A. (2011), Employee Stock Ownership as a Gift Exchange, Mid-year fellows symposium in honor of Louis Kelso, Rutgers University, 8-11 December. www.smlr.rutgers.edu/eso-giftexchange-presentation

Perroux F. (1942), Communauté, Presse Universitaire de France.

Perroux F. (1947), L'Alliance de l'Exigence Abstraite et de l'Exigence Expérimentale dans l'Economie Positive : une Leçon de l'Euvre de G. Pirou, Revue d'Economie Politique, $\mathrm{n}^{\circ} 4$.

Perroux F. (dir.) (1947), La participation des salariés aux responsabilités de l'entreprise, Presse Universitaire de France, publications de l'ISEA.

Perroux F. (1954), Le Don: Sa Signification Economique dans le Capitalisme Contemporain, Diogène, vol. 6.

Perroux F. (1970), Propos de Départ, Economies et Sociétés, Hors-série sur « la Participation”, vol. 10, septembre.

Perroux F. (1970), "Pour Lier Promotion et Participation", Economies et Sociétés, Hors-série sur « la Participation $», \mathrm{n}^{\circ} 10$, septembre.

Perroux F. (1990), L'économie du XX ${ }^{\text {ème }}$ Siècle, Presse Universitaire de Grenoble.

Perroux F. (1994), Economie et société : contrainte, échange, don, Presse Universitaires de France, 1960. Repris dans "Pouvoir et économie généralisée", Presse Universitaire de Grenoble, 1994.

Perroux F. (1994), Pouvoir et économie généralisée, Presse Universitaire de Grenoble.

Putterman L. (2006), Reciprocity, Altruism and Cooperative Production, in Ythier J.-M., Kolm, S.-C., Handbook of the economics of giving, altruism and reciprocity, Palgrave Macmillan.

Rotemberg J. (2006), Altruism, reciprocity and cooperation in the workplace, in Ythier J.-M., Kolm, S.-C., Handbook of the economics of giving, altruism and reciprocity, Palgrave Macmillan.

Sen A. (1966), Labor Allocation in a Cooperative Enterprise, Review of Economic Studies, vol. 33, pp. 361-371.

Villey D. et Nême C. (1996), Petite histoire des grandes doctrines économiques, Litec éditions Génin.

Ythier J.-M. et Kolm S.-C. (2006), Handbook of the economics of giving, altruism and reciprocity, Palgrave Macmillan. 